\title{
Tumor grafts derived from patients with head and neck squamous carcinoma authentically maintain the molecular and histologic characteristics of human cancers
}

Shaohua Peng ${ }^{1}$, Chad J Creighton ${ }^{2,6,7}$, Yiqun Zhang², Banibrata Sen ${ }^{1}$, Tuhina Mazumdar ${ }^{1}$, Jeffery N Myers ${ }^{3,8}$, Adrian Woolfson ${ }^{5}$, Matthew V Lorenzi ${ }^{5}$, Diana Bell ${ }^{4}$, Michelle D Williams ${ }^{4}$ and Faye M Johnson ${ }^{1,8^{*}}$

\begin{abstract}
Background: The patient-derived xenograft (PDX) model is likely to reflect human tumor biology more accurately than cultured cell lines because human tumors are implanted directly into animals; maintained in an in vivo, three-dimensional environment; and never cultured on plastic. PDX models of head and neck squamous cell carcinoma (HNSCC) have been developed previously but were not well characterized at the molecular level. HNSCC is a deadly and disfiguring disease for which better systemic therapy is desperately needed. The development of new therapies and the understanding of HNSCC biology both depend upon clinically relevant animal models. We developed and characterized the patient-derived xenograft (PDX) model because it is likely to recapitulate human tumor biology.
\end{abstract}

Methods: We transplanted 30 primary tumors directly into mice. The histology and stromal components were analyzed by immunohistochemistry. Gene expression analysis was conducted on patient tumors and on PDXs and cell lines derived from one PDX and from independent, human tumors.

Results: Five of 30 (17\%) transplanted tumors could be serially passaged. Engraftment was more frequent among HNSCC with poor differentiation and nodal disease. The tumors maintained the histologic characteristics of the parent tumor, although human stromal components were lost upon engraftment. The degree of difference in gene expression between the PDX and its parent tumor varied widely but was stable up to the tenth generation in one PDX. For genes whose expression differed between parent tumors and cell lines in culture, the PDX expression pattern was very similar to that of the parent tumor. There were also significant expression differences between the human tumors that subsequently grew in mice and those that did not, suggesting that this model enriches for cancers with distinct biological features. The PDX model was used successfully to test targeted drugs in vivo.

Conclusion: The PDX model for HNSCC is feasible, recapitulates the histology of the original tumor, and generates stable gene expression patterns. Gene expression patterns and histology suggested that the PDX more closely recapitulated the parental tumor than did cells in culture. Thus, the PDX is a robust model in which to evaluate tumor biology and novel therapeutics.

Keywords: Patient-derived xenograft, Translational animal models, Gene expression, Head and neck cancer

\footnotetext{
* Correspondence: fmjohns@mdanderson.org

'Departments of Thoracic/Head and Neck Medical Oncology, Unit 432, The University of Texas MD Anderson Cancer Center, 1515 Holcombe Boulevard, Houston 77030-4009, TX, USA

${ }^{8}$ The University of Texas Graduate School of Biomedical Sciences at Houston, Houston, TX, USA

Full list of author information is available at the end of the article
} 


\section{Background}

Approximately 52,610 new cases of head and neck cancer are diagnosed in the United States each year and worldwide annual incidence is estimated at 644,000 $[1,2]$. While locoregional control in advanced head and neck squamous cell carcinoma (HNSCC) has improved, recurrence is still common. Even when curative therapy is available, HNSCC is often a disabling and disfiguring cancer that can have a profound impact on important functions such as eating, speaking, sight, and hearing. These insults are compounded by distortions in facial appearance. To advance therapy for HNSCC, better laboratory models are needed to study HNSCC biology, systemic therapy, and radiotherapy.

There are several existing animal models for head and neck cancers [3,4]. No one model is ideal, and each has its advantages and disadvantages. These include chemicalinduced cancer models; syngeneic murine cancer cells injected back into immunocompetent mice from the same strain; transgenic mice expressing mutant KRAS [5,6] or activated $A K T$ with $p 53$ loss [7]; and xenograft models in which human HNSCC cell lines, grown on plastic in tissue culture, are injected into immunocompromised mice, either subcutaneously or into an orthotopic site such as the tongue or the floor of the mouth [8]. The orthotopic xenograft model is appealing because it uses human cancer cells in an appropriate anatomical site, is reliable, and recapitulates, to some extent, human tumor behavior. However, a major disadvantage of models that rely on cells grown on plastic is that these HNSCC cell lines have gene expression profiles that are markedly different from HNSCC tumors from patients [9].

An increasingly promising xenograft model, the patientderived xenograft (PDX), is developed by surgically implanting tumor tissue directly from a patient into an immunocompromised mouse. The resulting heterotransplanted tumors maintain the histologic characteristics of the primary tumor [10-13], and the pattern of response to chemotherapy resembles those observed in the clinic [14-17]. PDXs of non-small cell lung cancer (NSCLC) were shown to maintain the gene expression patterns of the original tumor [18]. Furthermore, the PDX model utilizes tumors from several individuals, suggesting that this approach could serve as a better surrogate for therapeutic studies in human HNSCC.

The purpose of our study is to generate HNSCC PDXs and characterize how well the model recapitulates human disease. Our hypothesis is that human HNSCC tumor tissue transplanted directly into nude mice maintains the molecular and histologic features of the original tumors. An additional unanswered question is the origin of the stromal components observed in PDX models. We transplanted 30 human HNSCC tumors directly into mice and serially transplanted those that engrafted. The histology was compared in the parental and PDX tumors. The origin of the stromal components was analyzed using mouse- and human- specific antibodies. Gene expression analysis was conducted on patient tumors and on PDXs and cell lines. This is the first published study of an HNSCC PDX model that has been characterized at the molecular level.

\section{Methods \\ HNSCC patient tumor engraftment into mice}

Residual tumor was taken at the time of surgery from 26 previously untreated patients undergoing definitive surgery and 4 patients undergoing surgical salvage for HNSCC. Upon arrival in the pathology suite, these tissues were transported immediately to the animal facility in sterile RPMI medium. For samples measuring $<0.5 \mathrm{~cm}^{3}$, the entire sample was implanted into a single nude mouse. For samples measuring $>0.5 \mathrm{~cm}^{3}$, the original patients' tumors (F0 generation) were divided; part of the tissue was implanted in a mouse, and the remaining portion was snap-frozen in liquid nitrogen or stored in RNAlater (Life Technologies, Carlsbad, CA). The tumor tissue used for implantation was minced into $2-\mathrm{mm}^{3}$ pieces, which were implanted subcutaneously into the flanks of anesthetized 6-week-old $\mathrm{Nu} / \mathrm{Nu}$ female mice that were bred onsite at MD Anderson. When the resulting tumors grew to $1 \mathrm{~cm}^{3}$, each tumor (F1 generation) was resected and divided as for the primary tumor and passaged into 5 mice (F2 generation). The process was repeated to produce subsequent generations. The tumors and the derived PDXs were named human oral squamous carcinoma HOSC 1-30; all PDX models maintained the same HOSC number as the parent tumor from which they were derived. All animal studies were performed in accordance with the policies of the Institutional Animal Care and Use Committee and were approved by the Institutional Review Board of The University of Texas MD Anderson Cancer Center.

\section{Cell culture and lines established from xenografts}

At the time of xenograft passage, remaining viable tumor tissue was reduced to 1 - to $2-\mathrm{mm}^{3}$ fragments, which were transferred to Dulbecco modified Eagle medium containing 10\% FBS, $100 \mathrm{U} / \mathrm{ml}$ penicillin, and $100 \mu \mathrm{g} / \mathrm{ml}$ streptomycin (Sigma, St. Louis, MO) and incubated at $37^{\circ} \mathrm{C}$ in an atmosphere containing $5 \% \mathrm{CO}_{2}$. The medium was renewed twice weekly once the cells had become attached. For 3D cell culture, the BioAssembler 3D culture system (n3D Biosciences, Inc., Houston, TX) was adopted according to the manufacturer's instructions [19]. Established HNSCC cell lines (Tu167 and Osc19) were maintained as previously described [20]. 


\section{Histologic characterization}

Tumor tissues from parent tumors and PDXs were formalin fixed, paraffin-embedded and stained with hematoxylin and eosin. The tumors were examined under light microscopy by a head and neck pathologist (M.D.W.). Tumors were evaluated for the degree of differentiation (formation of keratin, cytologic features, and growth pattern), presence of perineural invasion, desmoplastic stroma and extent of inflammation. The patient's surgical resection was also microscopically evaluated for lymph node metastases and presence or absence of extranodal extension.

\section{Immunohistochemistry}

Immunohistochemical (IHC) analysis was performed as described previously $[21,22]$. Briefly, $5-\mu \mathrm{m}$, paraffin-embedded tumor sections were deparaffinized, rehydrated, and subjected to antigen retrieval in sodium citrate buffer ( $\mathrm{pH}$ 6.0). Slides were quenched in $3 \% \mathrm{H}_{2} \mathrm{O}_{2}$ for $15 \mathrm{~min}$, rinsed in PBS, blocked in avidin for $10 \mathrm{~min}$, rinsed in PBS, blocked in biotin for $10 \mathrm{~min}$, and washed and blocked in whole serum for $15 \mathrm{~min}$. Slides were incubated with primary antibody (human specific anti-vimentin [Biocare Medical, Concord, CA] or human/mouse-specific antivimentin [Thermo Scientific, Hanover Park, IL]) or humanspecific keratin 5/6 (DAKO, Glostrup, Denmark) or PCNA (Biocare Medical) for $30 \mathrm{~min}$, rinsed in PBS, incubated in biotinylated anti-mouse IgG, rinsed in PBS, then incubated in streptavidin-horseradish peroxidase. The chromagen 3amino-9-ethylcarbozole (AEC) or 3,3'-diaminobenzidine (DAB) were used to detect antigen. Slides were counterstained with hematoxylin. Nuclear PCNA expression was quantified in 20 fields per sample using a 3-value intensity: 0 , none; low (weak to moderate); and high (strong).

\section{Gene expression array}

RNA was extracted from parent tumors, xenografts, and both PDX-derived and established HNSCC cell lines using an RNAeasy mini kit (Qiagen, Valencia, CA). The integrity of the RNA from each sample was measured by using the RNA 6000 Nano LabChip and a 2100 Bioanalyzer (Agilent Technologies, Palo Alto, CA). The quality and concentration of RNA was assessed on an ND-1000 spectrophotometer (NanoDrop, Wilmington, DE). The Affymetrix (Santa Clara, CA) GeneChip U133 Plus 2.0 was used without RNA amplification. Hybridization, washing, staining, and scanning were performed by Asuragen, Inc. (Austin, TX) as previously described [23]. All samples were run in a single batch to avoid batch effects.

Gene array data were quantile normalized; differential gene expression was assessed by two-sided $t$-test and is expressed as fold-change (using log-transformed data). Hierarchical clustering trees were generated by Eisen Cluster software [24]. Heat maps were generated by JavaTreeView [25]. Array data have been deposited in the Gene Expression Omnibus (GEO; accession GSE45153).

\section{Drug treatment of mice}

HOSC1-F7 tumors from 8 mice were passaged into 40 nude mice using the techniques described above. Once the tumors reached approximately $0.5 \mathrm{~cm}^{3}$ the mice were stratified for tumor size by TM into 4 groups which were then randomly assigned one of 4 treatment regimens by a researcher blinded to the stratification (SP). Dasatinib (20 mg/kg), BMS911543 (10 mg/kg), both, or vehicle was administered by oral gavage daily for 16 days. Dasatinib was purchased from the clinical pharmacy and BMS911543 was provided by the BristolMyers Squibb Company. Mice were killed 2 hours following the last drug dose, tumors were dissected, and the mice examined for distant metastases. The tumors were fixed and subjected to histological and IHC analysis as described previously [26].

\section{Real time PCR}

We extracted mRNA from HOSC1-F0 and HOSC1-F3 tumors using the Qiagen RNeasy minikit; synthesized cDNA using the Promega RT-PCR system; applied primers designed using Primer-Blast; and performed RTPCR using the SYBR Green chemistry as we previously described [27]. Expression of the L32 gene was used as an internal control.

\section{Results}

\section{Generation of patient-derived HNSCC xenografts}

We received 30 residual tumor samples from surgically resected specimens. Thirteen of the tissue samples were small $\left(<0.5 \mathrm{~cm}^{3}\right)$ and were implanted in their entirety, whereas 17 larger tumors $\left(>0.5 \mathrm{~cm}^{3}\right)$ were divided to allow RNA/DNA collection (Figure 1). The tumors were predominantly oral squamous cancer, because these are most likely to undergo primary surgical resection. Five of the implanted tumors engrafted, for an overall engraftment rate of $17 \%$. Selected patient characteristics are included in Additional file 1: Table S1.

\section{Poorly differentiated tumors with positive nodes were more likely to engraft}

Engraftment was more common among poorly differentiated and node-positive tumors (differences not significant by chi-squared test), but was not associated with patient clinical outcome (recurrence) or T stage (Table 1). Of the 8 poorly differentiated tumors, 3 engrafted (38\%); 2 of the 19 that were moderately differentiated engrafted (9\%); and none of the 3 that were well differentiated engrafted. Of the $13 \mathrm{~N} 0$ tumors, 1 engrafted (8\%), while 4 of the $13 \mathrm{~N}+$ tumors engrafted (31\%). None of the 4 tumors derived 


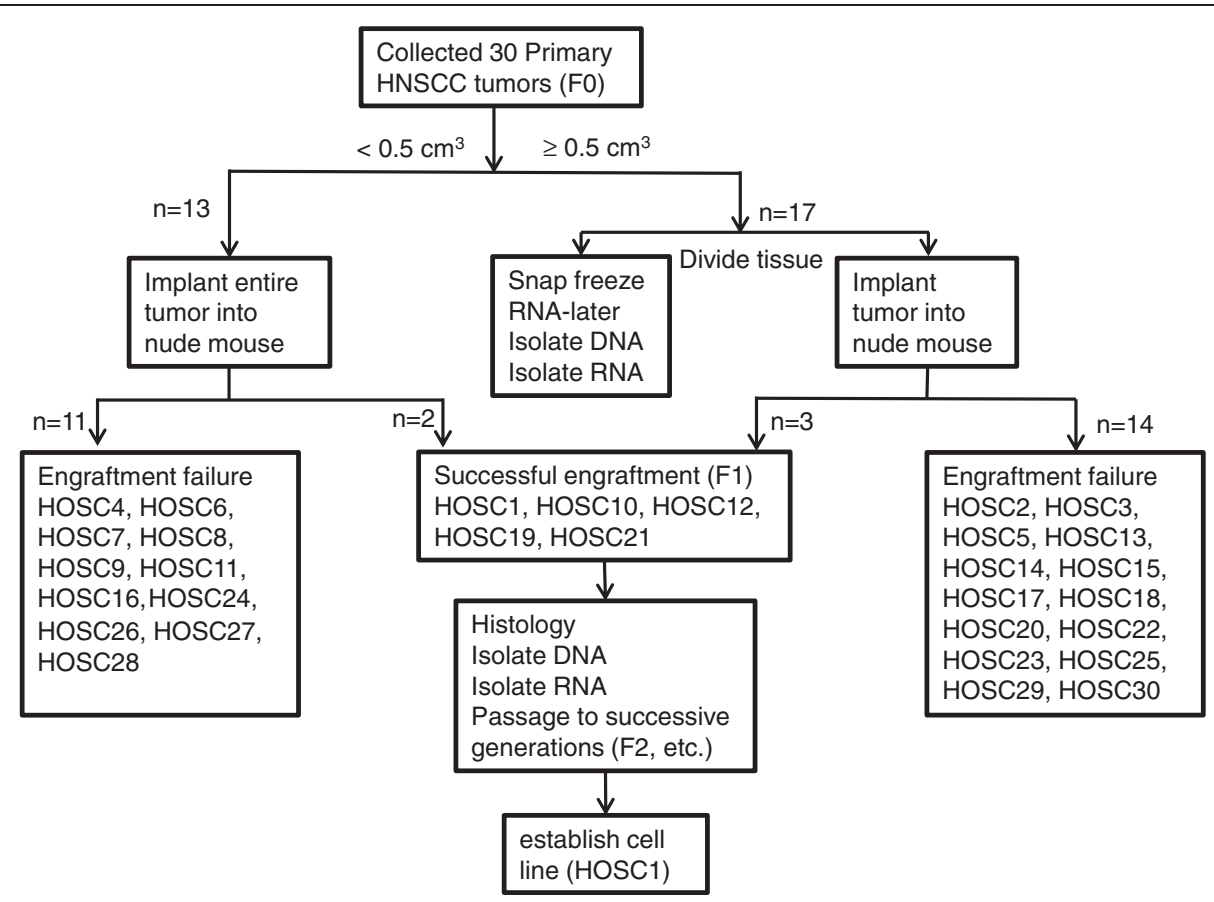

Figure 1 Flow sheet for the development of patient-derived xenografts and cell lines from parent head and neck cancer tissue specimens.

from recurrent tumors engrafted. Gene expression analysis of tumors that engrafted and those that did not revealed 992 probes whose expression was distinctly different between the two groups $(\mathrm{P}<0.01$, fold-change $>1.4)$ and that reflected diverse functions (Additional file 2: Figure S1 and Additional files 3, 4).

Table 1 Clinical and pathological characteristics of transplanted tumors

\begin{tabular}{llccc}
\hline & & Total & Engrafted & Percent engrafted \\
\hline Differentiation & Poor & 8 & 3 & 38 \\
& Moderate & 19 & 2 & 11 \\
& Well & 3 & 0 & 0 \\
Extracapsular & Present & 8 & 2 & 25 \\
Extension & Absent & 12 & 3 & 25 \\
\multirow{3}{*}{ Perineural } & No nodes & 10 & 0 & 0 \\
Invasion & Present & 11 & 3 & 27 \\
T Stage & Absent & 19 & 2 & 11 \\
& T1/T2 & 8 & 2 & 25 \\
\multirow{3}{*}{ N Stage } & T3/T4 & 18 & 3 & 17 \\
& Recurrent & 4 & 0 & 0 \\
& No & 13 & 1 & 8 \\
Recurrence & N1-3 & 13 & 4 & 0 \\
& Recurrent & 4 & 0 & 15 \\
\hline
\end{tabular}

Patient-derived xenografts maintained the histologic characteristics of the tumor from which they were derived

Patient-derived xenografts and the original human tumors were examined for histology, degree of differentiation, and presence of inflammation, perineural invasion, and necrosis (Additional file 1: Table S2). In all cases, the histology (invasive squamous carcinoma) and degree of differentiation of the PDX matched that of the parent tumor (Figure 2). There was a general trend for the PDX tumors to lose stroma and become more solid and homogeneous in successive generations. Mouse nerves were present for evaluation in 2 PDX tumors (HOSC10 and HOSC12), allowing evaluation for perineural invasion, which matched that of the parent tumor.

\section{Patient-derived xenografts lost human stromal components}

To determine whether the stroma observed in PDX tumors was of mouse or human origin, we utilized two antivimentin antibodies--one specific to human vimentin and a second that binds both human and mouse vimentin. Vimentin is a mesenchymal marker that stains fibroblasts and a fraction of squamous tumors that have undergone epithelial to mesenchymal transition [28]. No stromal staining for human vimentin was observed, however stroma did stain with the antibody which recognized both human and mouse (Figure 3, Additional file 1: Table S3, and Additional file 5: Figure S2). Only 2 of the PDXs had 


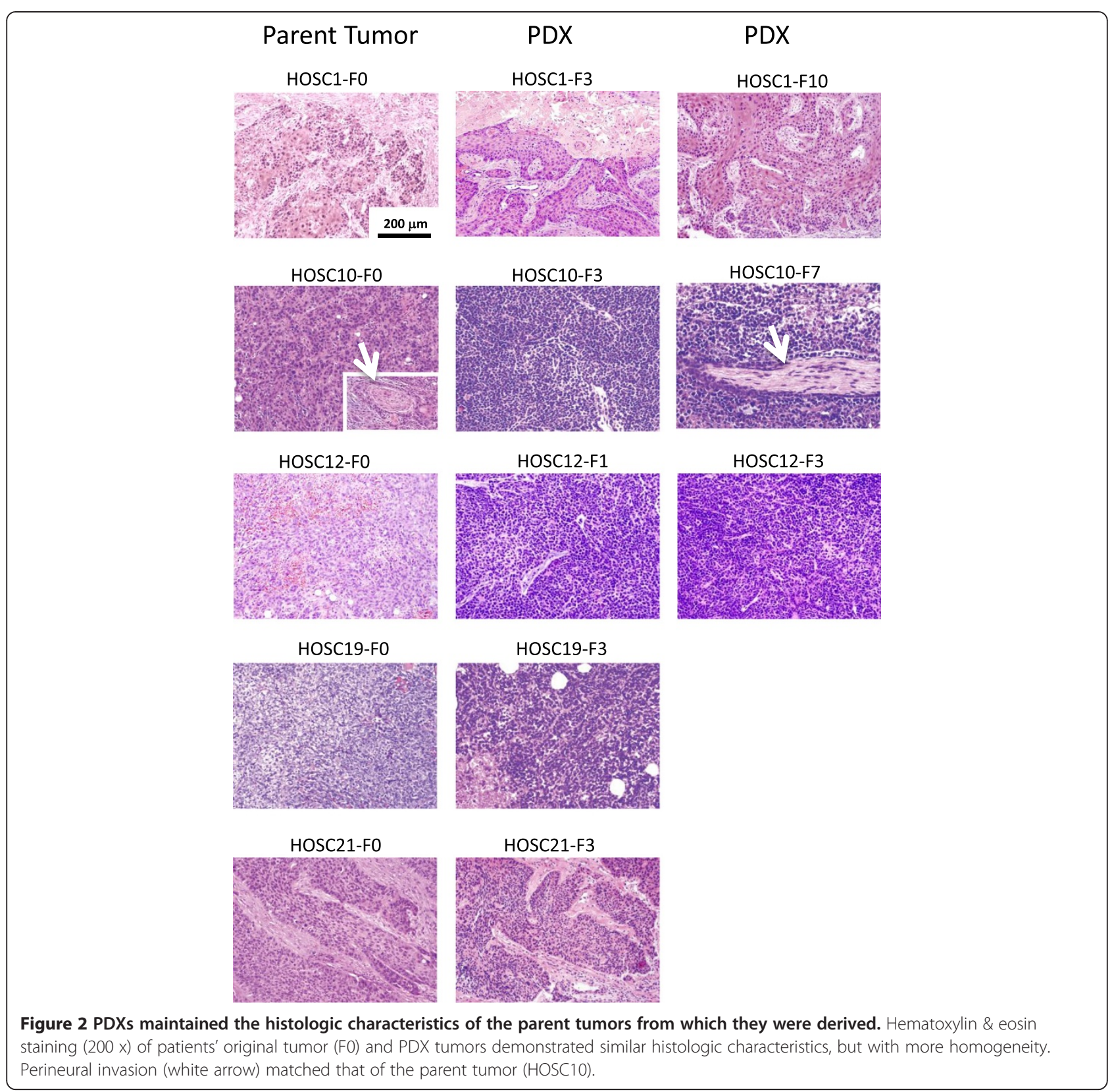

significant mouse stroma present; one had moderate mouse stroma, and the other 2 had only sparse mouse vessel staining. As expected, the human tumor cells in some of the PDX models stained weakly with vimentin. As a control we stained the PDX tumors for a human specific epithelial marker (keratin 5/6). As expected, there was no stromal staining. The tumor cell staining intensity varied widely between models (Additional file 6: Figure S3).

The growth rate of the 5 PDX models varied from model to model and the tumors tended to grow more quickly in subsequent generations (Additional file 7: Figure S4). The acceleration in growth rate is consistent with our finding that the cancer cell content of the tumors increased over time.

Gene expression patterns of patient-derived xenografts and parent tumors were distinct from those of human cancer cells in culture

Many HNSCC mouse models utilize cancer cells grown in culture, but HNSCC cell lines have gene expression profiles that are markedly different from those of patient tumors [9]. We performed unsupervised clustering of gene expression (on the basis of 54,000 gene transcript probes represented in the dataset) from all the human 


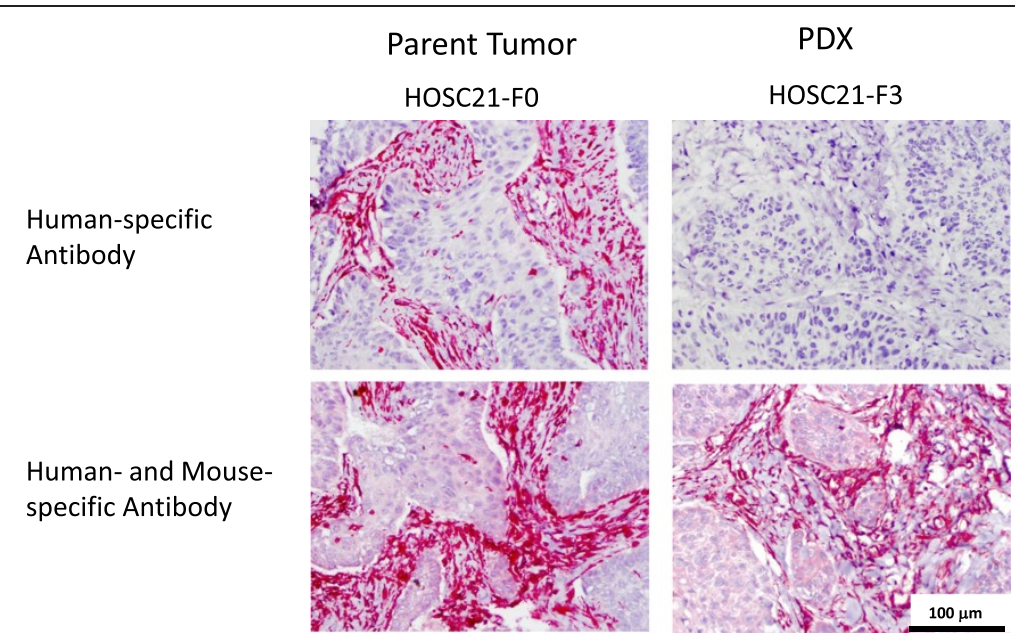

Figure 3 PDX tumors contained exclusively murine stromal components. Representative IHC is shown. Parental human tumors (F0) and PDXs (F3) were stained with anti-vimentin antibodies that are specific for human vimentin (top row) or both human and mouse vimentin (bottom row).

parent tumors, PDXs, a cell line derived from HOSC1 PDX, HOSC1 grown under 3D conditions, and 2 established HNSCC cell lines (Figure 4). As expected, all the cell lines clustered tightly together and were distinct from the tumor samples. Likewise, we found 1377 gene probes that were different between HNSCC parent tumors and HNSCC human cell lines in culture $(\mathrm{P}<0.01$, fold-change $>3$ ). In contrast, the PDX expression pattern

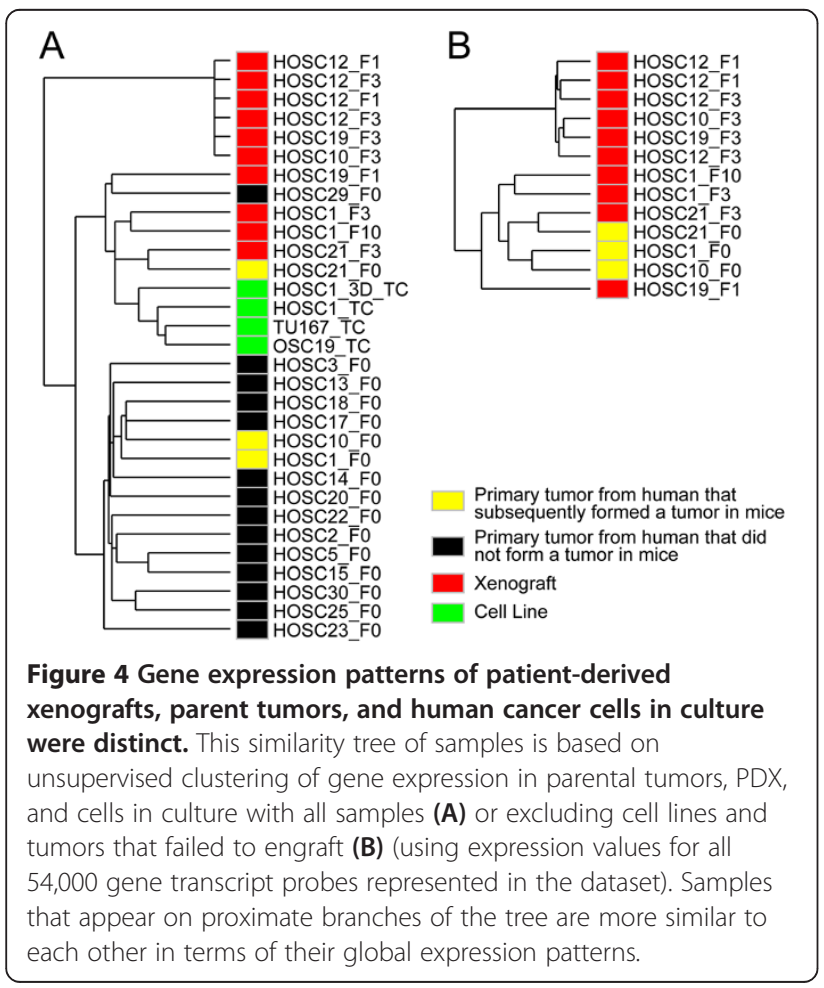

for these 1377 probes was quite similar to that of the human parent tumors (Figure 5A).

The majority of parent tumors clustered separately from the PDXs. Of the 3 matched pairs, one (HOSC21) clustered tightly with its xenograft, whereas the other 2 (HOSC1 and HOSC10) did not. No clinical or pathologic characteristics explained the better maintenance of gene expression in HOSC21 than in the other PDXs. The tight clustering of 2 generations of HOSC12 (F1 and F3) and HOSC1 (F3 and F10) demonstrated that gene expression was stable once the tumors were established in mice, although HOSC19 F1 and F3 did not cluster together.

Examining specific genes, we found 627 probes that were different between the PDX tumors and the parent tumors when only the 3 paired samples were analyzed $(\mathrm{P}<0.01$, fold-change $>1.4$ ) (Figure $5 \mathrm{~B}$ ). When all samples were analyzed, there were 936 distinct probes (Figure 5C). For genes with known functions, Additional files 8 and 9 list the gene ontogeny terms for genes with distinct expression. These include genes involved in cell adhesion and immune response. We chose 4 genes with distinct expression patterns between the parent tumor and the PDX model and confirmed expression differences using qPCR (Additional file 10: Figure S5).

\section{The PDX model of HNSCC can be used to test novel therapeutics}

Based on our previously published work, we hypothesized that JAK and Src inhibitors would have synergistic antitumor effects in vivo [26]. Mice bearing HOSC1 PDX tumors were treated with the Src inhibitor dasatinib, the JAK2 inhibitor BMS911543, both drugs, or vehicle control. Tumors were measured twice weekly and stained for PCNA to measure proliferation at the end of treatment. 

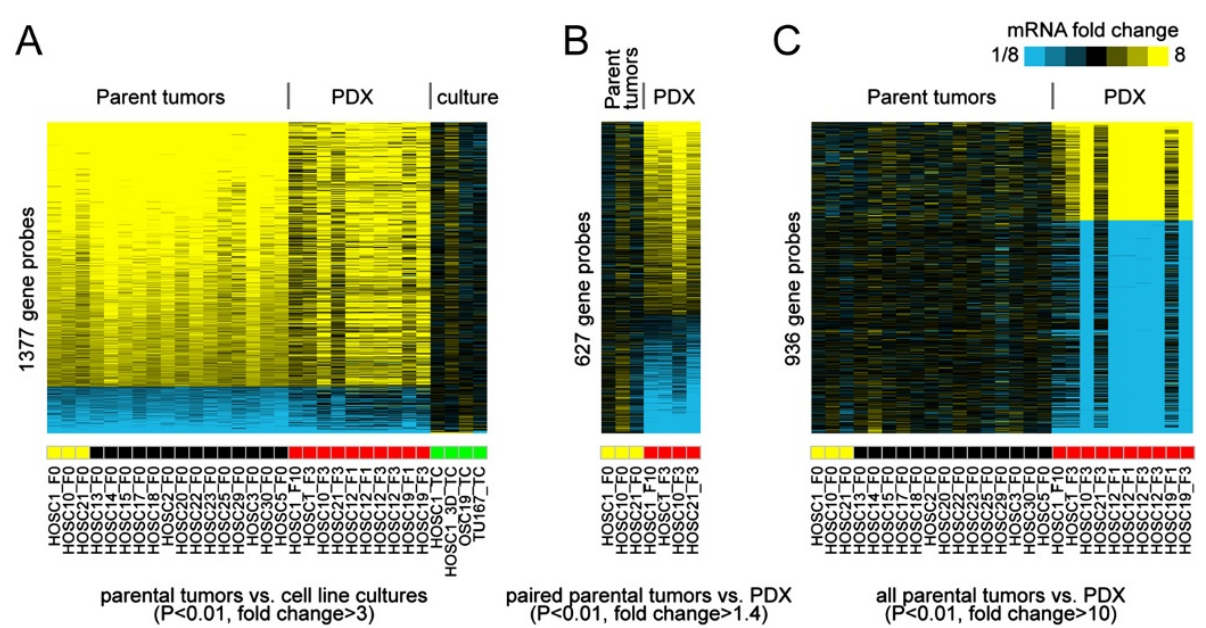

Figure $\mathbf{5}$ Gene expression patterns of patient-derived xenografts and parent tumors were distinct from that of human cancer cells in culture. (A) Analysis of gene expression data from parental tumors and human HNSCC cancer cells in culture revealed 1377 probes that were distinct $(P<0.01$, unpaired $t$-test, fold-change $>3$ in either direction). (B) Comparison of 3 parental tumors to the paired PDXs revealed 627 probes that were distinct $(P<0.01$, paired $t$-test, fold-change $>1.4)$. (C) Comparison of all parental tumors to all PDXs revealed 936 probes that were distinct $(P<0.01$, unpaired $t$-test, fold-change $>10)$. Specific gene ontogeny groups are listed in Additional files 3, 4. For each heat map, genes are centered on a given reference group to show relative expression differences.

By day 16 , growth of the tumors in the combination group had been inhibited by 63\% (Figure 6A). Likewise, there was a significant decrease in PCNA staining intensity consistent with decreased proliferation (Figure 6B). Specifically, the percent of cells staining with low, intermediate, and high intensity was 10,41 , and $49 \%$ in the control tissues and 54, 29, and 16\% in the tissues from mice treated with the combination therapy respectively.

\section{Discussion}

In this study we transplanted 30 human HNSCC tumors directly into mice and serially transplanted those that engrafted. The overall engraftment rate was $17 \%$ with poorly differentiated and node-positive tumors more likely to engraft. There were significant expression differences between the human tumors that subsequently grew in mice and those that did not. The tumors maintained the

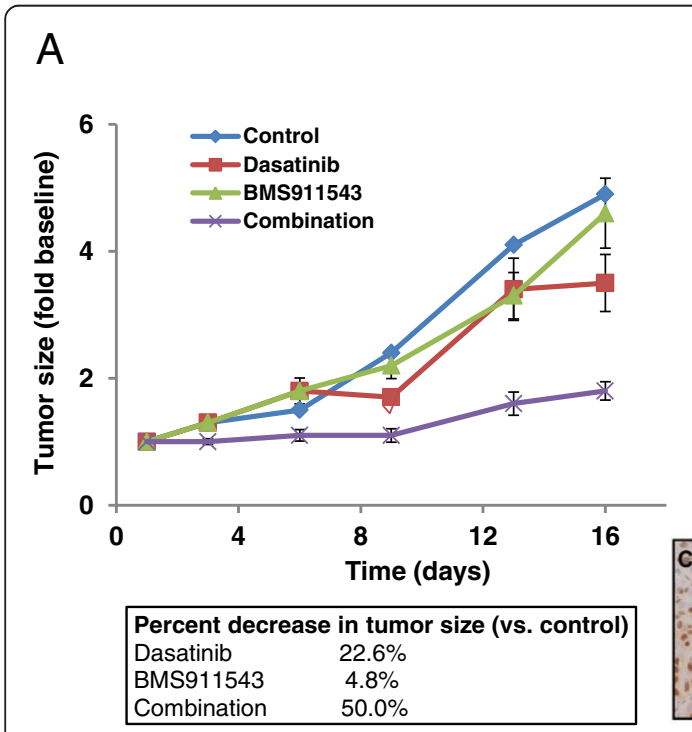

B

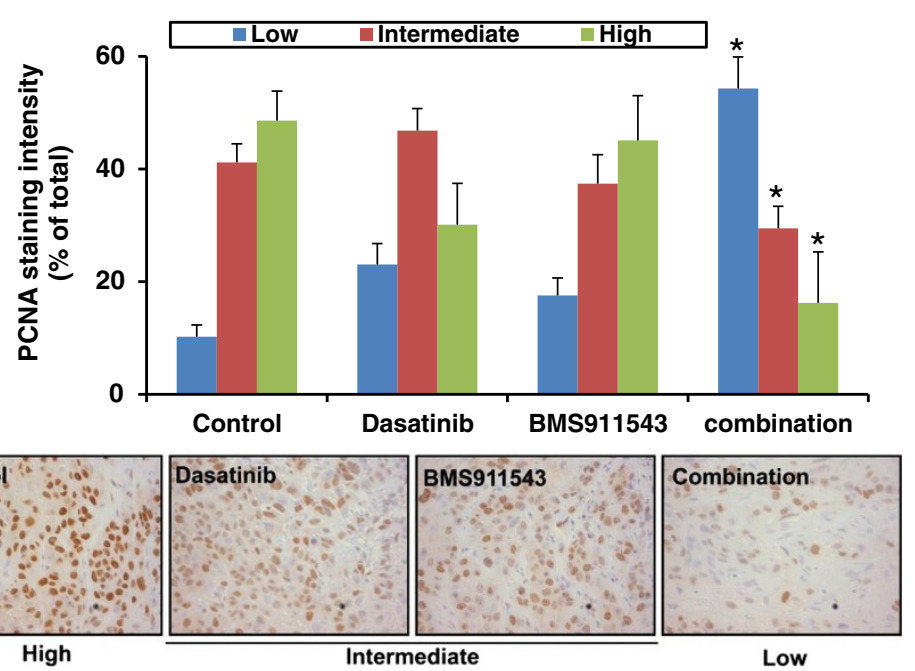

Figure 6 The combination of Src and JAK inhibition decreases in vivo proliferation compared to single agents. Nude mice bearing HOSC1 PDX tumors were treated with Dasatinib (20 mg/kg), BMS911543 (10 mg/kg), both, or vehicle for 16 days as indicated. (A) Tumor size was measured twice a week. (B) Tumors were stained for PCNA at the end of treatment and nuclear staining intensity scored and graphed. Representative IHC fields are shown. ${ }^{*} p<0.05$ compared to control (vehicle treated). 
histologic characteristics of the parent tumor, but were more homogeneous in successive generations. Human stromal components were lost upon engraftment. Gene expression between the PDXs and parent tumors were distinct, but stable in successive generations. The PDX expression pattern for gene probes that were different between parent tumors and human cell lines was quite similar to that of the human parent tumors.

Several PDX models have been developed and characterized, with several common themes emerging. In nearly all cases the histology of the original parent tumor was similar to that of the matched PDX [10-13]. This similarity persisted up to at least passage 12 [15]. In our study, tumors from passage 10 were histologically similar to parent tumors, with a loss of stroma over subsequent generations. Genetic abnormalities in the parent tumor such as specific mutations and gene copy number generally have been maintained in xenografts. In a study of colon cancer xenografts, there was concordance between gene copy number and mutation (NRAS, KRAS, BRAF, PIK3CA) in first- and second-generation xenografts and in the parent tumor [15]. Lung cancer models demonstrated similar karyotype and gene copy number as parent tumors by array comparative genomic hybridization (aCGH) [10].

The engraftment rate has varied greatly in different models, and rates ranging from $11 \%$ to $90 \%$ have been reported $[11,29]$. The highest engraftment rate was attributed to rigorous selection of viable cancer tissue and the relatively greater vascularity of the renal site of engraftment than of the usual subcutaneous site [16], although a subcutaneous colon cancer model had a similar engraftment rate of 87\% [15]. Engraftment correlated with less tumor differentiation [11,30], advanced stage [13], and poor donor prognosis [12,29,31,32] in most, but not all, studies [11,33]. In the best characterized HNSCC PDX model before ours, the engraftment rate was $11 \%$ overall. In that model, poorly differentiated tumors, larger tumors, recurrent tumors, and tumors from lymph node metastases were more likely to form xenografts [30,33]. Additional PDX models of HNSCC have been developed but have not been well characterized at the molecular level and most also demonstrated low engraftment rates [34-40]. In our study, tumors that were poorly differentiated and node positive were significantly more likely to engraft.

Factors other than differentiation and metastasis to lymph nodes may also influence engraftment rates. Tumors derived from metastatic sites have generally been more likely to engraft than those from primary sites [15,30], but not always [29]. Surprisingly, cold ischemic time did not influence engraftment [15]. Characteristics that predicted engraftment in NSCLC included squamous histology, poor differentiation, larger tumor size, and KRAS mutation [12]. Likewise, in pancreatic cancer the tumors that grew in mice were more likely to have SMAD4 mutations and an independently developed metastatic gene signature [31,41]. Efferth et al. examined 20 proteins in NSCLCs that were transplanted into mice and found that increased expression of JUN, FOS, cyclin D, and CDK4 predicted successful engraftment [42]. Our results show that tumors that engrafted had a gene expression profile that was distinct from the profile of those that did not engraft.

Few studies have rigorously examined stroma in PDX models. One of the most comprehensive characterizations of the PDX model showed that, after the first passage, tumor cells were enriched in the xenografts compared to the parent tumors, but the ratio of stroma:tumor was constant in subsequent passages. Human stroma was lost in the early passages [15]. In a breast cancer model, human stroma was replaced by mouse stroma after engraftment and tumor cells were enriched in the xenografts [29]. When NSCLC tumors were engrafted into NOD-scid IL2 $\gamma^{\text {null }}$ (NSG) mice, tumor-derived human leukocytes were maintained for up to 9 weeks in the first generation [43]. Similarly, we found that in our HNSCC PDX models, the human stroma was replaced by mouse stroma that was progressively less abundant in subsequent passages.

In the subcutaneous PDX models, metastases have not been described. However, nodal and distant (lung and liver) metastases have developed from orthotopic injection, in patterns consistent with clinical observations in colorectal cancer patients [15]. In a breast cancer model, both local nodal and distant metastases were found at rates that varied from $38 \%$ to $100 \%$ [29].

Several studies have compared gene expression in PDXs with that in the original parent tumors in lung, breast, and colon cancers. These studies show mixed results which are similar to ours; we found that the expression patterns of the PDX and parent tumors were distinct in 2 cases, but one parent tumor and PDX pair did cluster very tightly together. Unsupervised hierarchical clustering of 17 human NSCLC tumors and their matched heterotransplants revealed that 9 of the 17 animal tumors clustered with the primary tumors from which they were derived; 5 of the 8 primary tumors that did not cluster with their matched heterotransplants contained less than $10 \%$ tumor tissue [18]. In a breast cancer model, using expression of a set of 1291 intrinsic genes and unsupervised clustering, all xenografts and parent tumor pairs clustered together [29]. Unsupervised clustering of about $40 \mathrm{pa}$ tient tumors with matched early- and late-stage xenografts separated parent tumors from xenografts, but there were no major differences between early- and late-stage xenografts. Ontogeny analysis of genes that were differentially expressed between tumors and xenografts revealed genes encoding for extracellular matrix components and immune modulators consistent with the loss of human stroma in the xenografts [15]. Gene 
expression analysis in breast cancer xenografts demonstrated that the majority of probes were not differentially expressed between the parent tumor and its xenograft. In this study, gene ontology analysis demonstrated that the genes that were differentially expressed corresponded to the stromal compartment [17]. Likewise, an independent group identified stromal and extracellular matrix genes as differentially expressed in xenografts and parent tumors [44].

Gene expression was analyzed in 3 small cell lung cancer (SCLC) biopsy specimens, primary xenografts derived from the tumors, cell lines derived from the xenografts, and those same lines reimplanted into mice. When compared to normal lung, the tumors, xenografts, and cell lines had an expression pattern that was specific for SCLC. However, the gene expression patterns changed when the cancer cells were grown on plastic, and this expression pattern did not fully recover when the lines were subsequently re-injected in vivo (i.e., secondary xenografts). The expression patterns of the primary xenografts more closely resembled those of the parental tumors than those of the cell lines or the secondary xenografts [45]. Likewise, in our model, the genes that were expressed differently in cell lines grown in plastic vs. parental tumors were maintained in PDX tumors, suggesting that the PDX model more closely reflects the biology of the parental tumor than do cell lines.

The ultimate goal of developing a PDX model is to recapitulate tumor biology for the development of effective cancer therapies. When xenografts are treated with therapeutic agents used in patients with that tumor, similar response rates are usually observed, suggesting that the PDX model is superior to cell culture models in vitro or in vivo for drug development. When mice bearing colorectal cancer xenografts were treated with cetuximab, the response rates mirrored those seen in clinical trials of cetuximab, and tumors bearing KRAS mutations were resistant to cetuximab, as are most KRAS-mutant colon cancers in human patients [14]. Similar results were demonstrated in a separate group of xenografts derived from colorectal cancer in which irinotecan and cetuximab as single agents had response rates in the population of xenografts that were similar to those found in human populations [15]. When mice with NSCLC xenografts were treated with chemotherapy regimens that are commonly administered to NSCLC patients, response rates were similar to those in patients [16]. Fifteen colorectal cancer xenografts responded to cytotoxic chemotherapy agents that are active in colon cancer in human patients [17].

Data linking drug sensitivity in specific patients to their paired xenografts are sparse. In a pancreatic cancer model, there was a positive correlation between responses of the xenografts to gemcitabine and time to progression for the corresponding 7 patients who were treated with gemcitabine [31]. The most comprehensive and only prospective published study established xenografts from 14 patients with advanced metastatic disease and screened the tumors for sensitivity to 63 drugs. Two of the patients' xenografts were not sensitive to any drugs tested, but the other 12 patients underwent therapy based on the xenograft data. In 9 of the 12 patients, the cancer responded as predicted by this screening, while the two cancers whose xenografts were resistant did not respond to standard therapy [46]. Although these numbers are too small for rigorous statistical analysis, the correlation is striking and clearly indicates that the PDX model may be useful to predict drug sensitivity prospectively. We successfully utilized the HOSC1 model to determine the in vivo efficacy of JAK and Src inhibition, demonstrating that our model may be useful for developing novel cancer therapeutics [26].

The biggest limitations of our study were the low engraftment rate and lack of adequate tissue for analysis of all parental tumors. Many resected HNSCC tumors are small, and the residual tissue after standard pathologic analysis is completed is not adequate for research studies. The use of NSG rather than the Nude mouse may improve the engraftment rate and allow for the retention of human fibroblasts and lymphocytes [47-49]. Nonetheless, our study is the most comprehensive analysis and development of the PDX model for HNSCC to date.

\section{Conclusions}

We have demonstrated that developing a PDX model for HNSCC is feasible, although challenging because of the uncertain availability of adequate tumor. The engraftment rate for HNSCC was low, but it may be enhanced by using the NSG mouse, selecting patients with nodal disease and poorly differentiated tumors. Several common themes for PDX tumors were recapitulated by our work: the loss of human stroma; the fidelity of the histology; and the utility for testing therapeutic agents.

\section{Additional files}

Additional file 1: Table S1. Characteristics of patients and implanted tumors. Table S2. Histologic characteristics of parent tumors and patient-derived xenografts. Table S3. Immunohistochemical analysis with species-specific anti-vimentin antibodies in PDX tumors

Additional file 2: Figure S1. Human tumors that engrafted in mice had gene expression profiles distinct from those of human tumors that did not engraft.

Additional file 3: Heterotransplants that grew in mice vs. those that did not. Heterotransplants that grew in mice/those that did not.

Additional file 4: Heterotransplants that grew in mice vs. those that did not. Heterotransplants that grew in mice/those that did not.

Additional file 5: Figure S2. Human tumors that engrafted in mice had gene expression profiles distinct from those of human tumors that did not engraft.

Additional file 6: Figure S3. PDX tumor cells stained for a human-specific epithelial marker. PDXs were stained with anti-keratin 5/6 antibodies that are specific for human keratin using $\mathrm{HC}$. The stroma did not stain and the 
intensity of staining for the tumor cells varied widely between the different PDX models.

Additional file 7: Figure S4. The growth rates of the PDX models accelerated over time. When the implanted parental tumors grew to $1 \mathrm{~cm}^{3}$, it was resected and minced into $2-\mathrm{mm}^{3}$ pieces, which were implanted subcutaneously into mice (F1 generation). The process was repeated to produce subsequent generations. The time for a tumor to reach $1 \mathrm{~cm}^{3}$ reflects the growth rate of that tumor.

Additional file 8: Heterotransplant in mice as a group vs. original tumors in humans. Heterotransplant in mice as a group/original tumors in humans.

Additional file 9: Heterotransplant in mice as a group vs. original tumors in humans. Heterotransplant in mice as a group/original tumors in humans.

Additional file 10: Figure S5. Gene expression of 4 genes in the array demonstrates the same pattern of expression change when confirmed by individual qPCR. We extracted total RNA from HOSC1-F0 and HOSC1F3 specimens and measured gene expression levels using qPCR for 4 genes that had distinct expression on the array. Two genes that were up regulated on the array (GRIA1, MMP16) were similarly increased in the PDX model as compared to the parental tumor. Likewise, 2 genes that were down regulated in the array (HLA-DRA, LYZ) also demonstrated decreased expression by qPCR.

\section{Abbreviations}

(HNSCC): Head and neck squamous cell carcinoma; (PDX): Patient-derived xenograft; (NSCLC): Non-small cell lung cancer; (aCGH): Aarray comparative genomic hybridization; (SCLC): Small cell lung cancer; (HOSC): Human oral squamous carcinoma; (PCNA): Proliferating cell nuclear antigen; (NSG): NODscid IL2 $2 \gamma^{\text {nulll}} ;(\mathrm{IHC})$ : Immunohistochemical.

\section{Competing interests}

AW is an employee of Bristol-Myers Squibb which produces dasatinib and BMS911543. The remaining authors declare that they have no competing interests.

\section{Authors' contributions}

FMJ conceived and designed the project, supervised all the experiments, and wrote the manuscript. SP, BS, and TM helped to design and performed all the animal and bench experiments. CJC and $Y Z$ performed the gene expression analysis and prepared the corresponding figures. JM, DB, and MDW assisted with the study design and tissue acquisition, critically reviewed the data, and reviewed the manuscript. Additionally MDW performed all the histologic tissue analyses including interpretation of the $\mathrm{IHC}$ and prepared the corresponding figures. AW and $\mathrm{ML}$ were involved with the pre-clinical development of BMS-911543 and assisted with the study design and preparation of the manuscript. All authors gave final approval of the manuscript.

\section{Acknowledgments}

This work was supported by the National Cancer Institute (R01-CA143369-01). The immunohistochemical analysis and tissue processing were supported by $\mathrm{NCl}$ Cancer Center Core Support Grant CA16672. We thank Kathryn L. Hale for scientific editing and Brenda Robinson for help with manuscript preparation.

\section{Author details}

'Departments of Thoracic/Head and Neck Medical Oncology, Unit 432, The University of Texas MD Anderson Cancer Center, 1515 Holcombe Boulevard, Houston 77030-4009, TX, USA. ²Dan L. Duncan Cancer Center, Baylor College of Medicine, Houston, TX, USA. ${ }^{3}$ Head and Neck Surgery, The University of Texas MD Anderson Cancer Center, Houston 77030-4009, TX, USA. ${ }^{4}$ Pathology, The University of Texas MD Anderson Cancer Center, Houston 77030-4009, TX, USA. ${ }^{5}$ Discovery Oncology, Bristol-Myers Squibb Company, Princeton, NJ, USA. ${ }^{6}$ Department of Medicine, Baylor College of Medicine, Houston, TX, USA. ${ }^{7}$ Bioinformatics and Computational Biology, The University of Texas MD Anderson Cancer Center, Houston, TX, USA. ${ }^{8}$ The University of Texas Graduate School of Biomedical Sciences at Houston, Houston, TX, USA.
Received: 5 April 2013 Accepted: 14 August 2013

Published: 27 August 2013

\section{References}

1. Siegel R, Naishadham D, Jemal A: Cancer statistics, 2012. CA Cancer J Clin 2012, 62:10-29.

2. Jemal A, Bray F, Center MM, Ferlay J, Ward E, Forman D: Global cancer statistics. CA Cancer J Clin 2011, 61:69-90.

3. Smith LP, Thomas GR: Animal models for the study of squamous cell carcinoma of the upper aerodigestive tract: a historical perspective with review of their utility and limitations. Part A. Chemically-induced de novo cancer, syngeneic animal models of HNSCC, animal models of transplanted xenogeneic human tumors. Int I Cancer 2006, 118:2111-2122.

4. Thomas GR, Smith LP: Animal models for the study of squamous cell carcinoma of the upper aerodigestive tract: a historical perspective with review of their utility and limitations part B. Transgenic mouse models. Int J Cancer 2006, 118:2379-2380.

5. Caulin C, Nguyen T, Longley MA, Zhou Z, Wang XJ, Roop DR: Inducible activation of oncogenic K-ras results in tumor formation in the oral cavity. Cancer Res 2004, 64:5054-5058.

6. Raimondi AR, Vitale-Cross L, Amornphimoltham P, Gutkind JS, Molinolo A: Rapid development of salivary gland carcinomas upon conditional expression of K-ras driven by the cytokeratin 5 promoter. Am J Pathol 2006, 168:1654-1665.

7. Moral M, Segrelles C, Lara MF, Martinez-Cruz AB, Lorz C, Santos M, Garcia-Escudero R, Lu J, Kiguchi K, Buitrago A, et al: Akt activation synergizes with Trp53 loss in oral epithelium to produce a novel mouse model for head and neck squamous cell carcinoma. Cancer Res 2009, 69:1099-1108.

8. Myers JN, Holsinger FC, Jasser SA, Bekele BN, Fidler IJ: An orthotopic nude mouse model of oral tongue squamous cell carcinoma. Clin Cancer Res 2002, 8:293-298.

9. Chung CH, Parker JS, Karaca G, Wu J, Funkhouser WK, Moore D, Butterfoss D, Xiang D, Zanation A, Yin X, et al: Molecular classification of head and neck squamous cell carcinomas using patterns of gene expression. Cancer Cell 2004, 5:489-500

10. Cutz JC, Guan J, Bayani J, Yoshimoto M, Xue H, Sutcliffe M, English J, Flint J, LeRiche J, Yee J, et al: Establishment in severe combined immunodeficiency mice of subrenal capsule xenografts and transplantable tumor lines from a variety of primary human lung cancers: potential models for studying tumor progression-related changes. Clin Cancer Res 2006, 12:4043-4054.

11. Anderson TM, Hess SD, Egilmez NK, Nwogu CE, Lenox JM, Bankert RB: Comparison of human lung cancer/SCID mouse tumor xenografts and cell culture growth with patient clinical outcomes. J Cancer Res Clin Oncol 2003, 129:565-568.

12. John T, Kohler D, Pintilie M, Yanagawa N, Pham NA, Li M, Panchal D, Hui F, Meng F, Shepherd FA, Tsao MS: The ability to form primary tumor xenografts is predictive of increased risk of disease recurrence in early-stage non-small cell lung cancer. Clin Cancer Res 2011, 17:134-141.

13. Monsma DJ, Monks NR, Cherba DM, Dylewski D, Eugster E, Jahn H, Srikanth S, Scott SB, Richardson PJ, Everts RE, et al: Genomic characterization of explant tumorgraft models derived from fresh patient tumor tissue. J Trans/ Med 2012, 10:125.

14. Bertotti A, Migliardi G, Galimi F, Sassi F, Torti D, Isella C, Cora D, Nicolantonio FD, Buscarino MCP, et al: A molecularly annotated platform of patient-derived xenografts ("Xenopatients") identifies HER2 as an effective therapeutic target in Cetuximab-Resistant colorectal cancer. Cancer Discovery 2011, 1:508-523.

15. Julien S, Merino-Trigo A, Lacroix L, Pocard M, Goere D, Mariani P, Landron S, Bigot $L$, Nemati F, Dartigues $P$, et al: Characterization of a large panel of patient-derived tumor xenografts representing the clinical heterogeneity of human colorectal cancer. Clin Cancer Res 2012, 18:5314-5328.

16. Dong X, Guan J, English JC, Flint J, Yee J, Evans K, Murray N, Macaulay C, $\mathrm{Ng}$ RT, Gout PW, et al: Patient-derived first generation xenografts of non-small cell lung cancers: promising tools for predicting drug responses for personalized chemotherapy. Clin Cancer Res 2010, 16:1442-1451.

17. Reyal F, Guyader C, Decraene C, Lucchesi C, Auger N, Assayag F, De Plater L, Gentien D, Poupon MF, Cottu P, et al: Molecular profiling of patient-derived breast cancer xenografts. Breast Cancer Res 2012, 14:R11.

18. Fichtner I, Rolff J, Soong R, Hoffmann J, Hammer S, Sommer A, Becker M, Merk J: Establishment of patient-derived non-small cell lung cancer 
xenografts as models for the identification of predictive biomarkers. Clin Cancer Res 2008, 14:6456-6468.

19. Souza GR, Molina JR, Raphael RM, Ozawa MG, Stark DJ, Levin CS, Bronk LF, Ananta JS, Mandelin J, Georgescu MM, et al: Three-dimensional tissue culture based on magnetic cell levitation. Nature Nanotechnol 2010, 5:291-296.

20. Johnson FM, Saigal B, Tran H, Donato NJ: Abrogation of signal transducer and activator of transcription 3 reactivation after Src kinase inhibition results in synergistic antitumor effects. Clin Cancer Res 2007, 13:4233-4244.

21. Chaw SY, Majeed AA, Dalley AJ, Chan A, Stein S, Farah CS: Epithelial to mesenchymal transition (EMT) biomarkers-E-cadherin, beta-catenin, APC and Vimentin-in oral squamous cell carcinogenesis and transformation. Oral Oncol 2012, 48:997-1006.

22. Konig F, Krekeler G, Honig JF, Cordon-Cardo C, Fischer G, Korabiowska M: Relation between human papillomavirus positivity and p16 expression in head and neck carcinomas-a tissue microarray study. Anticancer Res 2007, 27:283-288

23. Chen C, Mendez E, Houck J, Fan W, Lohavanichbutr P, Doody D, Yueh B, Futran ND, Upton M, Farwell DG, et al: Gene expression profiling identifies genes predictive of oral squamous cell carcinoma. Cancer Epidemiol Biomarkers Prev 2008, 17:2152-2162.

24. Eisen MB, Spellman PT, Brown PO, Botstein D: Cluster analysis and display of genome-wide expression patterns. Proc Natl Acad Sci USA 1998, 95:14863-14868.

25. Saldanha AJ: Java Treeview-extensible visualization of microarray data. Bioinformatics 2004, 20:3246-3248.

26. Sen B, Peng S, Woods DM, Wistuba I, Bell D, El-Naggar AK, Lai SY, Johnson FM: STAT5A-mediated SOCS2 expression regulates Jak2 and STAT3 activity following c-Src inhibition in head and neck squamous carcinoma. Clin Cancer Res 2012, 18:127-139.

27. Saintigny P, Peng S, Zhang L, Sen B, Wistuba II, Lippman SM, Girard L, Minna JD, Heymach JV, Johnson FM: Global evaluation of Eph receptors and ephrins in lung adenocarcinomas identifies EphA4 as an inhibitor of cell migration and invasion. Mol Cancer Ther 2012, 11:2021-2032.

28. Dutsch-Wicherek M, Lazar A, Tomaszewska R, Kazmierczak W, Wicherek L: Analysis of metallothionein and vimentin immunoreactivity in pharyngeal squamous cell carcinoma and its microenvironment. Cell Tissue Res 2013, 352:341-349.

29. DeRose YS, Wang G, Lin YC, Bernard PS, Buys SS, Ebbert MT, Factor R, Matsen C, Milash BA, Nelson E, et al: Tumor grafts derived from women with breast cancer authentically reflect tumor pathology, growth, metastasis and disease outcomes. Nat Med 2011, 17:1514-1520.

30. Braakhuis BJ, Sneeuwloper G, Snow GB: The potential of the nude mouse xenograft model for the study of head and neck cancer. Arch Otorhinolaryngol 1984, 239:69-79.

31. Garrido-Laguna I, Uson M, Rajeshkumar NV, Tan AC, De Oliveira E, Karikari C, Villaroel MC, Salomon A, Taylor G, Sharma R, et al: Tumor engraftment in nude mice and enrichment in stroma- related gene pathways predict poor survival and resistance to gemcitabine in patients with pancreatic cancer. Clin Cancer Res 2011, 17:5793-5800.

32. Giovanella BC, Vardeman DM, Williams LJ, Taylor DJ, De Ipolyi PD, Greeff PJ, Stehlin JS, Ullrich A, Cailleau R, Slamon DJ, et al: Heterotransplantation of human breast carcinomas in nude mice. Correlation between successful heterotransplants, poor prognosis and amplification of the HER-2/neu oncogene. Int J Cancer 1991, 47:66-71.

33. Braakhuis BJ, Laarman DA, Nauta JJ, Van Walsum M, Van Dongen GA, Snow GB: Transplantability of human head and neck squamous cell carcinomas in athymic nude mice. Arch Otolaryngol Head Neck Surg 1989, 115:1076-1078.

34. Robbins KT, Rosenberg W, Weiss B, Varki NM: Growth characteristics of human laryngeal carcinoma in athymic mice. J Otolaryngol 1991, 20:117-122.

35. Wennerberg J: Changes in growth pattern of human squamous-cell carcinomas of the head and neck during serial passages in nude mice. Int J Cancer 1984, 33:245-250.

36. Wennerberg J, Trope C, Biorklund A: Heterotransplantation of human head and neck tumours into nude mice. Acta Otolaryngol 1983, 95:183-190.

37. Zatterstrom UK, Wennerberg J, Attewell R, Ask A: Squamous cell carcinoma of the head and neck heterotransplanted to nude mice: Take rate in relation to patient survival. Cancer 1990, 66:145-151.

38. Zatterstrom UK, Wennerberg J: Growth of xenografted head and neck cancer in nude mice pre-treated with whole body irradiation. Acta Otolaryngol 1991, 111:1170-1177.
39. Zatterstrom UK, Kallen A, Wennerberg J: Cell cycle time, growth fraction and cell loss in xenografted head and neck cancer. In Vivo 1991, 5:137-142.

40. Zatterstrom UK, Braakhuis BJ, Wennerberg J, Van Dongen GA, Attewell R, Nauta JJ, Laarman DA, Ask A: Growth of xenografted squamous cell carcinoma of the head and neck-possible correlation with patient survival. APMIS 1992, 100:976-980.

41. Ramaswamy S, Ross KN, Lander ES, Golub TR: A molecular signature of metastasis in primary solid tumors. Nat Genet 2003, 33:49-54.

42. Efferth T, Koomagi R, Mattern J, Volm M: Expression profile of proteins involved in the xenotransplantability of non-small cell lung cancers into athymic nude mice. Int J Oncol 2002, 20:391-395.

43. Simpson-Abelson MR, Sonnenberg GF, Takita H, Yokota SJ, Conway TF Jr, Kelleher RJ Jr, Shultz LD, Barcos M, Bankert RB: Long-term engraftment and expansion of tumor-derived memory $T$ cells following the implantation of non-disrupted pieces of human lung tumor into NOD-scid IL2Rgamma(null) mice. J Immunol 2008, 180:7009-7018.

44. Bergamaschi A, Hjortland GO, Triulzi T, Sorlie T, Johnsen H, Ree AH, Russnes HG, Tronnes S, Maelandsmo GM, Fodstad O, et al: Molecular profiling and characterization of luminal-like and basal-like in vivo breast cancer xenograft models. Mol Oncol 2009, 3:469-482.

45. Daniel VC, Marchionni L, Hierman JS, Rhodes JT, Devereux WL, Rudin CM, Yung R, Parmigiani G, Dorsch M, Peacock CD, Watkins DN: A primary xenograft model of small-cell lung cancer reveals irreversible changes in gene expression imposed by culture in vitro. Cancer Res 2009, 69:3364-3373.

46. Hidalgo M, Bruckheimer E, Rajeshkumar NV, Garrido-Laguna I, De Oliveira E, Rubio-Viqueira B, Strawn S, Wick MJ, Martell J, Sidransky D: A pilot clinical study of treatment guided by personalized tumorgrafts in patients with advanced cancer. Mol Cancer Ther 2011, 10:1311-1316.

47. Ito M, Hiramatsu H, Kobayashi K, Suzue K, Kawahata M, Hioki K, Ueyama Y, Koyanagi Y, Sugamura K, Tsuji K, et al: NOD/SCID/gamma(c)(null) mouse: an excellent recipient mouse model for engraftment of human cells. Blood 2002, 100:3175-3182.

48. Kim MP, Evans DB, Wang H, Abbruzzese JL, Fleming JB, Gallick GE: Generation of orthotopic and heterotopic human pancreatic cancer xenografts in immunodeficient mice. Nat Protoc 2009, 4:1670-1680.

49. Bankert RB, Balu-lyer SV, Odunsi K, Shultz LD, Kelleher RJ Jr, Barnas JL, Simpson-Abelson M, Parsons R, Yokota SJ: Humanized mouse model of ovarian cancer recapitulates patient solid tumor progression, ascites formation, and metastasis. PLOS One 2011, 6:e24420.

doi:10.1186/1479-5876-11-198

Cite this article as: Peng et al:: Tumor grafts derived from patients with head and neck squamous carcinoma authentically maintain the molecular and histologic characteristics of human cancers. Journal of Translational Medicine 2013 11:198.

\section{Submit your next manuscript to BioMed Central and take full advantage of:}

- Convenient online submission

- Thorough peer review

- No space constraints or color figure charges

- Immediate publication on acceptance

- Inclusion in PubMed, CAS, Scopus and Google Scholar

- Research which is freely available for redistribution 